\title{
Stepwise Progression of Centrosome Defects Associated with Local Tumor Growth and Metastatic Process of Human Pancreatic Carcinoma Cells Transplanted Orthotopically into Nude Mice
}

\author{
Masaki Shono, Norihiro Sato, Kazuhiro Mizumoto, Naoki Maehara, \\ Masafumi Nakamura, Eishi Nagai, and Masao Tanaka \\ Department of Surgery and Oncology, Graduate School of Medical Sciences, Kyushu University, Fukuoka, Japan
}

\begin{abstract}
SUMMARY: Recent evidence indicates that loss of centrosome integrity may be a major cause of genetic instability underlying various human cancers. The aim of this study was to define the role of centrosome defects during the in vivo tumor progression of pancreatic carcinoma using an orthotopic implantation model. Injection of Suit-2 human pancreatic cancer cells into the pancreata of nude mice reproduced the pattern of local tumor growth and distant metastasis observed in humans. Pancreatic xenografts, peritoneal disseminations, and hepatic metastases were harvested, and tumor cells were examined for centrosomes by immunofluorescence microscopy. Centrosome abnormalities, characterized by increased numbers of centrosomes, were detected in only a small fraction of parental Suit-2 cells in culture, whereas the frequency was markedly increased in cells isolated from the pancreatic xenografts. Abnormal centrosome numbers were found at higher frequencies in metastatic foci than in pancreatic xenografts. A significant positive correlation existed between the fraction of cells with multiple centrosomes and that with multipolar mitotic spindles, suggesting a functional involvement of aberrant centrosomes in spindle disorganization and chromosome missegregation. In addition, the increased frequency of abnormal centrosomes was associated with an enhanced degree of chromosomal instability. These findings suggest a novel model of pancreatic tumor progression whereby a stepwise increase in the magnitude of centrosomal abnormalities confers an increased chance for aberrant mitotic events, thus accelerating genetic instability and causing the tumor to progress to a more advanced stage. (Lab Invest 2001, 81:945-952).
\end{abstract}

$P$ ancreatic carcinoma is one of the most aggressive and treatment-refractory malignancies in humans, with an overall 5-year survival rate of less than $5 \%$ (Landis et al, 1999). In general, the clinical picture is characterized by the tumor's rapid spread to the surrounding vital organs and the frequent occurrence of distant metastases. Improving the prognosis of patients with such a deadly disease will require advances in our understanding of the molecular basis of its aggressive behavior and rapid progression.

Accumulating evidence suggests that multiple genetic alterations are necessary for the development and progression of pancreatic carcinoma (Hruban et al, 2000). For example, mutations of the K-ras oncogene and p53 tumor suppressor gene are frequently detected in pancreatic cancer (Pellegata et al, 1994). Although the mechanisms through which these mutations are generated are poorly understood, an underlying genetic instability may be required for multiple

\section{Received January 16, 2001.}

This work was supported in part by a grant from the Ministry of Education, Science, Sports, and Culture of Japan. NS is a research fellow of the Japan Society for the Promotion of Science.

Address reprint requests to: Dr. Kazuhiro Mizumoto, Department of Surgery and Oncology, Graduate School of Medical Sciences, Kyushu University, Fukuoka 812-8582, Japan.E-mail: mizumoto@mailserver. med.kyushu-u.ac.jp mutations to develop during a relatively short period (Loeb, 1991). Genetic instability is a defining feature of various human malignancies and has been considered to be a major driving force in multistep carcinogenesis (Lengauer et al, 1998). In fact, pancreatic cancer is frequently characterized by numerical and structural aberrations in chromosomes, a hallmark of genetic instability (Griffin et al, 1995; Hahn et al, 1995). Cytogenetic analyses of primary and metastatic pancreatic carcinomas have shown that chromosomal abnormalities occur at higher frequencies in metastatic tumors than in primary tumors (Zojer et al, 1998). Furthermore, pancreatic carcinomas xenografted in nude mice acquire a higher degree of genetic aberration during tumor dissemination (Reyes et al, 1996). These findings indicate that genetic instability may play a pivotal role, not only in pancreatic cancer development, but also in disease progression.

Recently, several lines of evidence have indicated that loss of centrosome integrity and the resulting mitotic defects may be important causes of genetic instability (Doxsey, 1998; Pihan and Doxsey, 1999; Zimmerman et al, 1999). The centrosome is a major microtubule organizing center in animal cells (Kellogg et al, 1994). Because the centrosome plays a critical role in assembly of bipolar mitotic spindles, an abnormal number of centrosomes can result in the formation of dysfunctional mitotic spindles and chromo- 
somal missegregation. Structural and functional abnormalities of centrosomes have been reported in various malignant tumors in humans (Carroll et al, 1999; Kuo et al, 2000; Lingle et al, 1998; Pihan et al, 1998). We reported previously that supernumerary centrosomes of various sizes and shapes are common features of pancreatic carcinomas (Sato et al, 1999). Thus, defects in centrosome function could be an underlying cause of genetic instability in pancreatic cancers, but the role of these abnormalities during tumor progression has yet to be determined.

It is difficult to examine centrosome dynamics during sequential progression of a given tumor in humans. However, orthotopic transplantation of human cancer cells into nude mice provides a model for clinical tumor spread, including extensive local tumor growth and distant metastases (Fu et al, 1992; Vezeridis et al, 1989). In the present study, we investigated changes in centrosome status during the progression of pancreatic carcinoma implanted in nude mice. In this system, we found a stepwise progression of centrosomal defects during local tumor growth and metastasis of xenografted tumors. Further, increased frequency of abnormal centrosomes was associated with enhanced chromosomal instability. These findings suggest an association between centrosome defects and pancreatic cancer progression and, thus, add a new dimension to our understanding of the molecular biology of this highly malignant neoplasm.

\section{Results}

\section{Establishment and Characteristics of Suit-2 Orthotopic Xenografts}

To establish the orthotopic implantation model, Suit-2 (human pancreatic carcinoma) cell suspension was injected into the pancreata of nude mice. All mice implanted with Suit-2 cells developed palpable tumors (100\% take rate) within 21 days after implantation (Table 1). The xenografted tumor (Fig. 1A), measuring 2 to $3 \mathrm{~cm}$ in diameter had extensively replaced the pancreas of each subject and, in most cases, invaded neighboring organs (spleen, stomach, and small bowel). In all mice, multiple dissemination nodules were observed throughout the peritoneal cavity, mainly in the mesentery (Fig. 1B). Liver metastases, recognized as white nodules on the periphery of the liver, were found in 7 of the 10 (70\%) mice (Fig. 1C). Macroscopically, tumors in the pancreas and metastatic sites were poorly differentiated adenocarcinomas proliferating mainly in a medullary fashion with many mitotic

Table 1. Local Tumor Growth and Distant Metastases Detected in Suit-2 Orthotopic Implantation Models

\begin{tabular}{lc}
\hline \multicolumn{1}{c}{ Tumor site } & Take rate $(\%)$ \\
$(n=10)$ \\
\hline Local tumor growth & $10 / 10(100)$ \\
Peritoneal dissemination & $10 / 10(100)$ \\
Liver metastasis & $7 / 10(70)$ \\
\hline
\end{tabular}
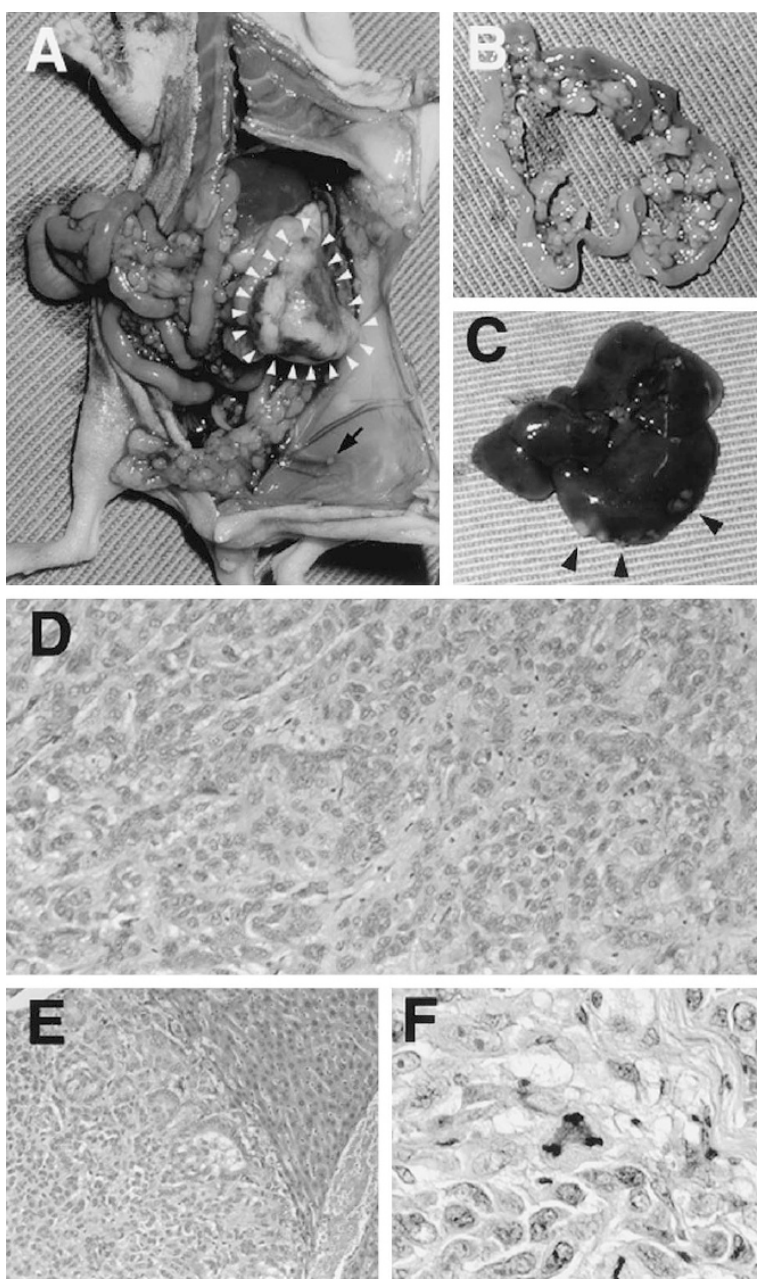

Figure 1.

Macroscopic and microscopic findings in an orthotopic implantation model of a human pancreatic cancer cell line, Suit-2. A, Extensive tumor growth (white arrowheads) in the pancreas of a nude mouse. A black arrow indicates a dissemination nodule on the peritoneum. B, Multiple dissemination nodules in the mesentery of a nude mouse. C, Metastatic tumors (arrowheads) on the periphery of a liver of a nude mouse. D, Histopathology of a pancreatic xenograft showing poorly differentiated adenocarcinoma. E, Histopathology of a hepatic metastasis. F, Aberrant mitotic cell in a tissue section from a peritoneal dissemination. The cell shows segregation of the chromosomes into three fragments.

figures (Fig. 1, D and E). The neoplastic cells showed nuclear pleomorphism, and nucleoli were prominent. Remarkably, some mitotic cells displayed abnormal alignment and/or segregation of the chromosomes (Fig. 1F), suggesting frequent occurrence of aberrant mitotic events.

\section{Changes in Centrosome Profile during Pancreatic Cancer Progression in an Orthotopic Implantation Model}

To investigate changes in centrosome profiles during pancreatic cancer progression, centrosome numbers were evaluated in cells isolated from pancreatic xenografts and metastatic tumors and compared with parental Suit-2 cells. For this purpose, indirect immunofluorescence microscopy was performed using antibodies against $\gamma$-tubulin and pericentrin, both of 
which are major components of centrosomes (Dictenberg et al, 1998; Doxsey et al, 1994; Stearns et al, 1991). The frequency of cells showing abnormal centrosome numbers (more than two centrosomes) was determined for each tumor sample obtained from the six mice (Table 2). A majority of the parental Suit-2 cells in culture showed normal (one or two) centrosome numbers (Fig. 2A), and the proportion of cells that contained abnormal centrosome numbers was only $3.2 \%$ (Fig. 3). In contrast, cells isolated from the xenografted tumors and metastases showed extensive centrosomal abnormality characterized by increased numbers of centrosomes (Fig. 2B). Sometimes, multiple centrosomes were organized into large patchy aggregates or linear structures (Fig. 2C). In pancreatic xenografts ( $n=6$ ), the proportion of cells showing abnormal centrosome numbers was increased to $18.3 \%$ (5.7-fold), which was significantly higher than the proportion in the parental cells $(p<$ 0.0001; Fig. 3). Furthermore, this number was even higher in peritoneal dissemination $(n=6)$ and liver metastases $(n=5)$, where $23.3 \%$ (7.3-fold) and 27.0\% (8.4-fold) of cells displayed abnormal centrosome numbers, respectively (Fig. 3). The proportion of cells with abnormal centrosome numbers in both peritoneal dissemination and liver metastases was significantly higher than that in pancreatic xenografts $(p=0.0346$ and $p=0.0014$, respectively). These findings indicate that stepwise progression of centrosome defects may be associated with local tumor growth and distant metastasis of pancreatic carcinoma xenografted in nude mice.

\section{Spindle Abnormalities and Chromosome Missegregation during Pancreatic Cancer Progression in an Orthotopic Implantation Model}

To evaluate the effect of abnormal centrosome duplication on spindle assembly during mitosis, we stained cells with an antibody to $\alpha$-tubulin. In parental cells grown in culture, nearly all mitotic cells displayed symmetric bipolar spindles where normal metaphase plates were established (Fig. 4, A and B). The mitotic cells in pancreatic xenografts and metastatic tumors, however, often exhibited atypical spindles organized by multiple poles (Fig. 4, C to F). Whereas the proportion of cells showing multipolar spindles was only
$4.9 \%$ of all metaphase cells in the parental cells, this number was increased to $27 \%$ (5.5-fold) in pancreatic xenografts, $34.9 \%$ (7.1-fold) in peritoneal dissemination, and $37.7 \%$ (7.7-fold) in liver metastases. Furthermore, a significant positive correlation existed between the fraction of cells with abnormal centrosome numbers and the fraction with multipolar mitotic spindles ( $p=0.0003$; Fig. 5), suggesting functional involvement of aberrant centrosomes in spindle disorganization. The multipolar spindle formation was associated with additional metaphase abnormalities, including asymmetrical alignment of the condensed chromosomes and unaligned chromosomal material (Figure 4, D and F). In addition, we found that these spindle defects sometimes resulted in unbalanced segregation of the chromosomes into more than two fragments (Fig. 6). This suggests that some cells with abnormal metaphase arrangements can progress through mitosis, thus increasing the risk of daughter cells with chromosome abnormalities.

\section{Relation between Centrosome Defects and Chromosome Instability in an Orthotopic Implantation Model}

To examine the relation between abnormal centrosome duplication and chromosomal instability during pancreatic cancer progression, we performed a fluorescence in situ hybridization (FISH) analysis with probes specific for chromosomes 1 and 8, which we used as marker chromosomes. We evaluated chromosome numbers by FISH rather than by conventional metaphase analysis so that cells could be studied at all phases of the cell cycle. The degree of chromosome instability was defined as the fraction of cells in which the chromosome number differed from the modal ploidy status. In parental cells, the rate of instability of chromosome 1 was $12.2 \%$, but this rate increased to $26.1 \%$ in pancreatic xenografts, $22.2 \%$ in peritoneal dissemination, and $23.7 \%$ in liver metastases (Fig. 7A). Although we found no difference in the instability rate between pancreatic xenografts and metastatic tumors, the rate in pancreatic xenografts was significantly higher than that in parental cells $(p=$ 0.0123). Similar results were obtained for chromosome 8 (Fig. 7B). Thus, the increase in the frequency of abnormal centrosomes during pancreatic tumor

Table 2. Frequency of Centrosome Abnormality in Pancreatic Xenografts and Metastatic Lesions in an Orthotopic Implantation Model ${ }^{a}$

\begin{tabular}{cccc}
\hline No. of animals & Pancreatic xenograft (\%) & Peritoneal dissemination (\%) & Liver metastasis (\%) \\
\hline 1 & 19.6 & 21.1 & ND \\
2 & 16.5 & 25.3 & 31.5 \\
3 & 19.9 & 25.8 & 29.2 \\
4 & 19.1 & 26.4 & 32.5 \\
5 & 13.7 & 21.2 & 18.3 \\
6 & 21.1 & 19.9 & 23.6 \\
mean $(\mathrm{SD})$ & $18.3(2.7)$ & $23.3(2.8)$ & $27.0(5.9)$ \\
\hline
\end{tabular}

ND, not determined.

${ }^{a}$ At least 200 cells in each sample were examined for centrosome numbers, and the percentage of cells with more than two centrosomes was determined. 

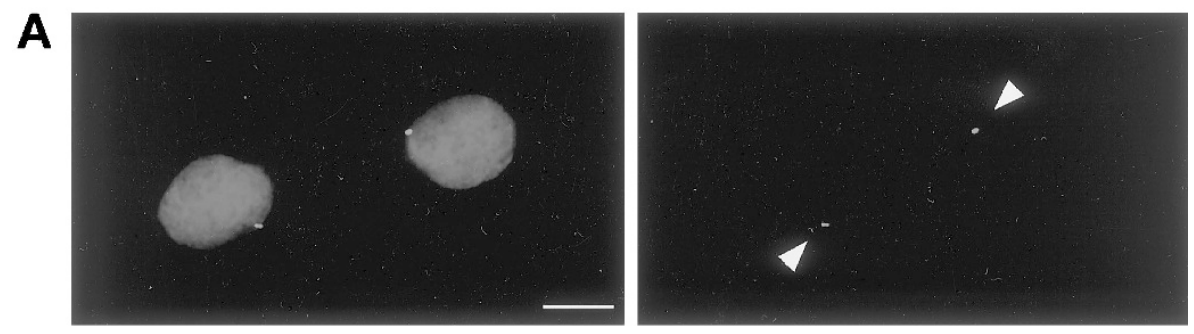

B
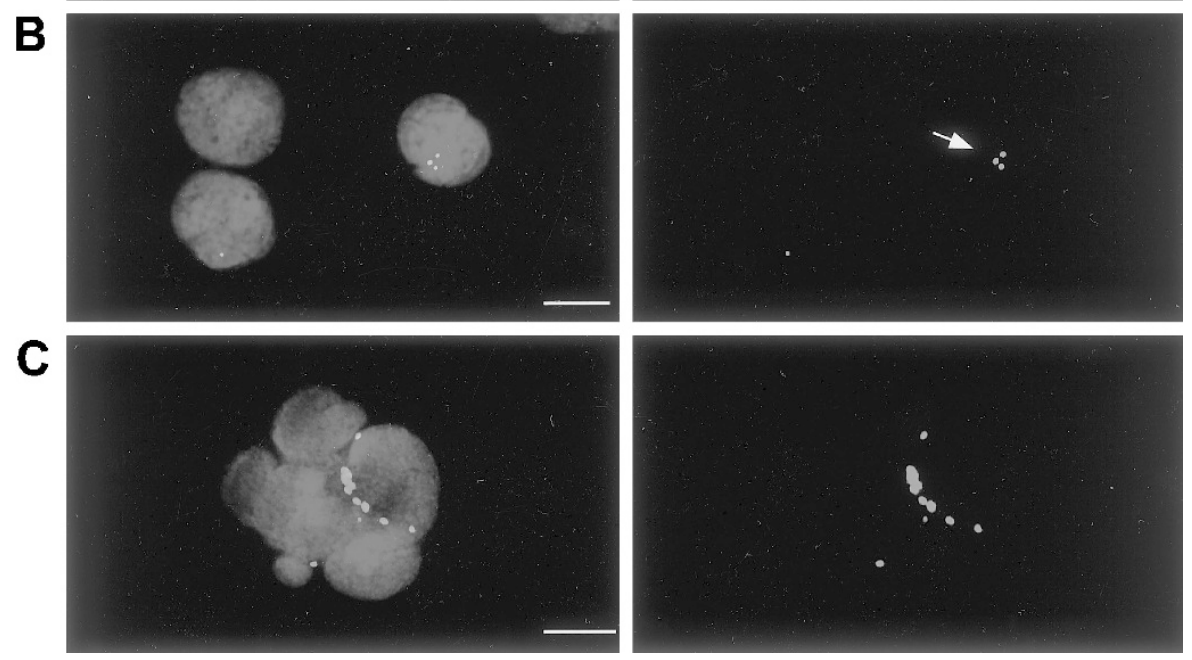

Figure 2.

Centrosome profiles of parental Suit-2 cells in culture and of cells isolated from pancreatic xenografts and metastatic tumors. Cells were stained with $\gamma$-tubulin antibody followed by Alexa 488-conjugated secondary antibody and propidium iodide. A, Parental cells showing one or two centrosomes (arrowheads) per cell. B, Cells isolated from a pancreatic xenograft. Cells contain three centrosomes (arrow). C, Cell isolated from a liver metastasis. The cell contains a highly lobulated nucleus and multiple centrosomes organized into a linear structure. Scale bars, $10 \mu \mathrm{m}$.

progression may be responsible for the enhanced degree of chromosome instability.

\section{Discussion}

In the current investigation, we attempted to define the role of centrosome-defects during the progression of pancreatic carcinoma growing orthotopically in nude mice. The orthotopic implantation of human pancreatic cancer cells into the pancreata of nude mice serves as a model that resembles the clinical features of tumor spread, including extensive local tumor growth and distant metastases. Using this model, we observed the following: (a) a stepwise increase in the frequency of cells showing abnormal centrosome numbers during local tumor growth and metastasis of xenografted tumors; (b) abnormally amplified centrosomes that contribute to the assembly of multipolar mitotic spindles, resulting in chromosome missegregation; and (c) an increase in the frequency of centrosome abnormality associated with increased numerical chromosome alterations. We suggest that the increasing number of centrosomal defects may play an essential role in the progression of pancreatic carcinoma possibly by maintaining or accelerating genetic instability. Our findings provide an explanation for the observation that numerous genetic alterations accumulate during a relatively short period of pancreatic tumor progression (Hilgers and Kern, 1999; Reyes et al, 1996).
We reported previously that centrosome abnormalities, characterized by an increased size and number of centrosomes and their irregular distribution, may develop at a relatively early stage of human pancreatic ductal carcinogenesis (Sato et al, 1999). In an experimental model, development of pancreatic cancer in transgenic mice expressing the simian virus 40 tumor antigen was associated with formation of multiple centrosomes (Levine et al, 1991). These observations provided evidence that centrosome defects contribute to early stages of pancreatic carcinogenesis. Our present results suggest that abnormal centrosome duplication is associated, not only with tumorigenesis, but also with progression of pancreatic carcinoma. In agreement with this model, an extensive analysis of a large series of biliary malignancies revealed that centrosome abnormality occurs at higher frequencies in the advanced stage than in the early stage (Kuo et al, 2000). The pattern of centrosome alterations in human pancreatic cancers at different stages has yet to be determined. An understanding of this pattern is necessary to establish the precise role of centrosomal abnormality in pancreatic cancer progression.

The present study demonstrates a clear difference in centrosome profiles between in vitro cultures and tumors grown in nude mice. In cells isolated from the pancreatic xenografts, we found a 5.7-fold increase in the proportion of cells with abnormal centrosome numbers. The mechanism responsible for this change in 


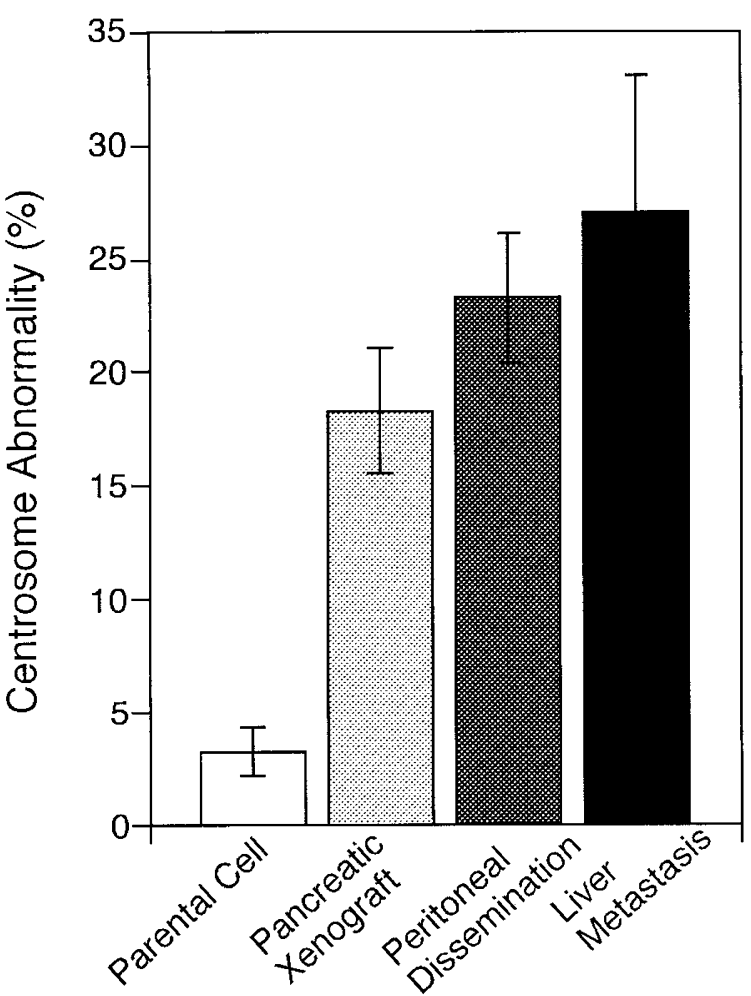

Figure 3.

Frequency of centrosome abnormalities in parental Suit-2 cells and cells isolated from pancreatic xenografts, peritoneal disseminations, and liver metastases. Percentages presented are the mean \pm SD (error bar) of three independent experiments (for parental cells) or six animals (for pancreatic xenografts, peritoneal disseminations, and liver metastases).

centrosome phenotype is unknown. The most likely explanation is that centrosome dysregulation may be a stress response of the cell to the tumor microenvironment, characterized by hypoxia, acidosis, and nutrient deprivation. In support of this idea, centrosome overduplication has been reported in response to cellular stresses, such as DNA damage caused by irradiation (Sato et al, 2000). Furthermore, previous reports have shown that the tumor microenvironment induces aneuploidy and genetic instability (Reynolds et al, 1996; Rofstad et al, 1996). We suggest that the tumor microenvironment leads to centrosome abnormality, which results in karyotypic heterogeneity within the tumor. However, it remains unclear whether microenvironmental stresses can affect centrosome duplication directly or modulate other components critical for the regulation of the centrosome duplication cycle.

During tumor progression, cells undergo dynamic changes in their chromosome composition that lead to the selection of cells adapted most effectively to the given environment. Cytogenetic analyses of primary and metastatic pancreatic carcinomas have shown that chromosome abnormalities occur at higher frequencies in metastatic tumors than in primary tumors (Zojer et al, 1998). Alterations in the numbers and structures of chromosomes reflect defective mitosis, including unequal distribution of chromosomes to daughter cells. In the present study, we have shown that abnormally amplified centrosomes can nucleate
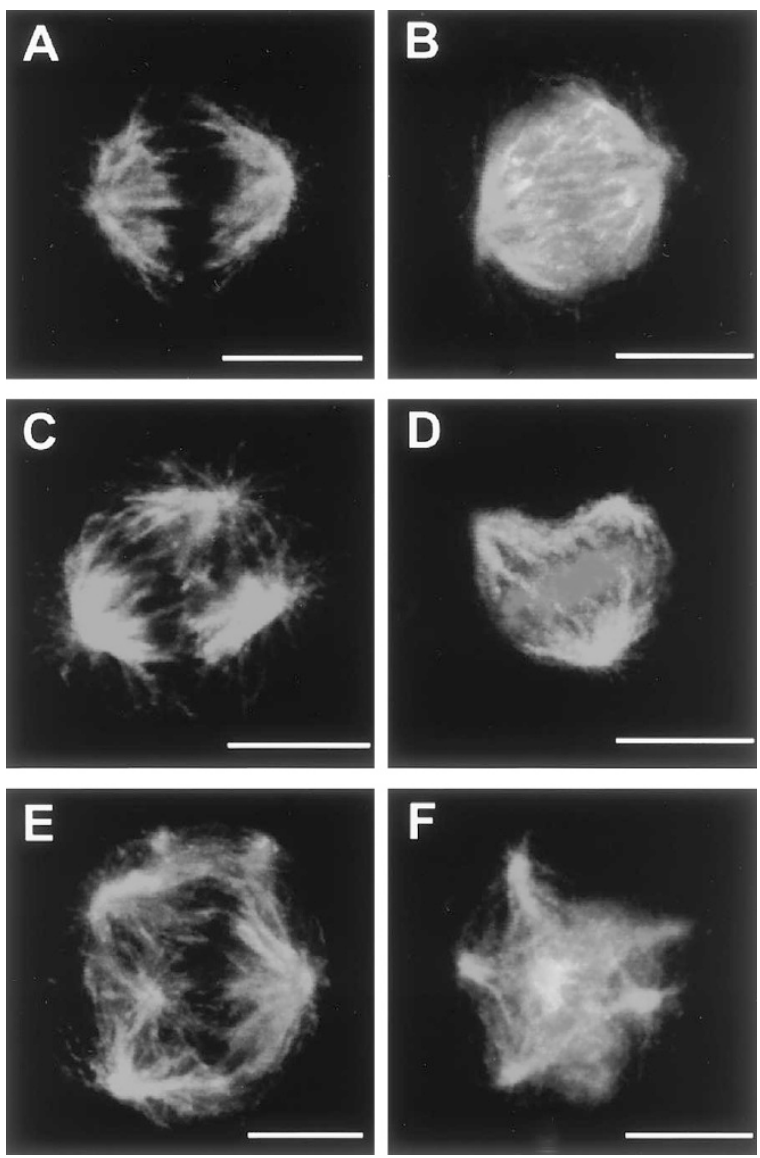

Figure 4.

Mitotic figures in parental Suit-2 cells and cells isolated from pancreatic xenografts and metastatic tumors. Cells were stained with $\alpha$-tubulin antibody followed by Alexa 488-conjugated secondary antibody and propidium iodide. A and $B$, Parental cells containing typical bipolar spindles. C and D, Cells isolated from the pancreatic xenograft showing tripolar spindles. $\mathrm{E}$ and $\mathrm{F}$, Cells isolated from liver metastases showing extensive mitotic defects characterized by multipolar spindle formation. Scale bars, $10 \mu \mathrm{m}$.

microtubules and function as spindle poles, leading to atypical multipolar mitoses. Consequently, the multidirectional forces generated by the multipolar spindles may lead to chromosomal breaks or missegregation of chromosomes into more than two progeny, thus producing cells with altered chromosomal structures and/or numbers. In fact, during local tumor growth, the increase in the number of chromosomal alterations corresponded to the increase in the frequency of centrosome abnormalities. Thus, our findings strongly suggest that centrosome defects may be a major cause of genetic instability.

In conclusion, our present results demonstrate a stepwise progression of centrosome abnormality during local tumor formation and metastasis of human pancreatic carcinoma cells orthotopically implanted into nude mice. The abnormal centrosome phenotype contributes to aberrant mitoses organized by multipolar spindles, thereby producing heterogeneous subpopulations of cells with altered chromosomal numbers and/or structures. The causative factor(s) affecting centrosome behavior during pancreatic cancer progression is unknown, but our findings raise the 


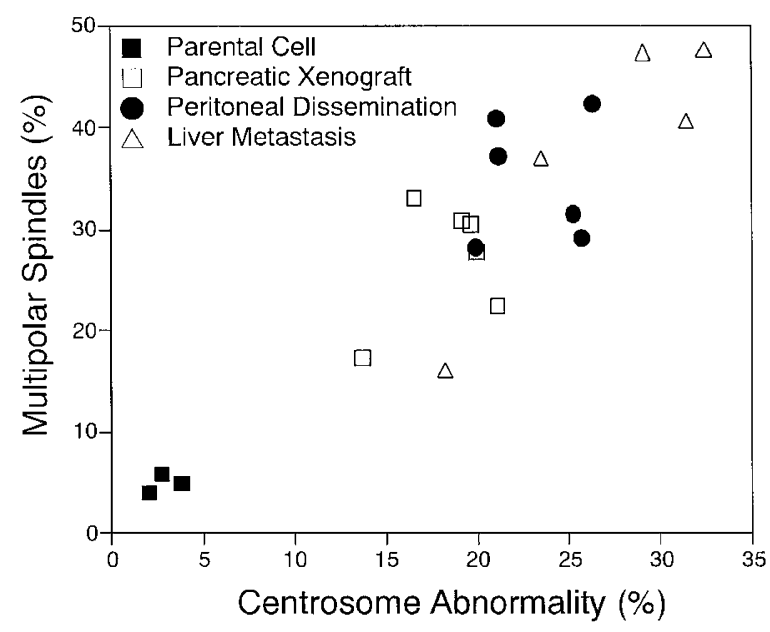

Figure 5.

Relation between centrosome abnormality and multipolar mitotic spindles in parental Suit-2 cells and cells isolated from pancreatic xenografts and metastatic tumors. A significant positive correlation exists between the frequency of centrosome abnormality and the frequency of multipolar mitotic spindles ( $p=0.0003$, Spearman's rank correlation).

possibility that centrosome abnormality plays a critical role in the genetic instability and rapid progression of pancreatic carcinoma.

\section{Materials and Methods}

\section{Cells and Culture Conditions}

We used a human pancreatic cancer cell line, Suit-2, established from a metastatic liver tumor of pancreatic ductal adenocarcinoma (Iwamura et al, 1987). Cells were maintained in DMEM (Sigma Chemical Company, St. Louis, Missouri) supplemented with 10\% fetal bovine serum (FBS), streptomycin $(100 \mu \mathrm{g} / \mathrm{ml})$, and penicillin $(100 \mathrm{U} / \mathrm{ml})$ at $37^{\circ} \mathrm{C}$ in a humidified atmosphere containing $10 \% \mathrm{CO}_{2}$.

\section{Orthotopic Implantation Model}

Six-week-old athymic nude mice (BALB/c nu/nu), weighing 15 to $19 \mathrm{~g}$, were obtained from Nippon SLC (Hamamatsu, Japan) and used to establish the orthotopic transplantation model. Suit-2 cells were suspended in serum-free DMEM at a concentration of $10^{7}$ cells/ml. Each mouse was anesthetized with ketamine (80 mg/Kg, ip) and xylazine (8 mg/Kg, ip), a left lateral laparotomy was made, and the spleen and distal pancreas were mobilized. The Suit- 2 cell suspension $\left(0.1 \mathrm{ml}\right.$, approximately $10^{6}$ cells) was injected directly into the body-tail of the pancreas. After implantation, the mice were inspected daily for any bleeding or wound complications and were checked for tumor formation three times a week by palpation. Palpable tumors were detected after 21 days in all mice, and the mice were killed when they were moribund (21 to 38 days after tumor implantation). Peritoneal and thoracic cavities were examined macroscopically for abnormalities including tumor formation. Representative specimens from the pancreas, liver, peritoneum, lung, and spleen were subjected to hematoxylin and eosin staining for histological examinations. In six cases, tumors from the pancreas and two metastatic sites (peritoneum and liver) were excised under sterile conditions and placed immediately in a complete medium to prepare for immunofluorescence microscopy or $\mathrm{FISH}$ analysis.

\section{Cell Preparation and Indirect Immunofluorescence Microscopy}

Tissues from the pancreatic xenografts and metastatic tumors were minced into approximately $1-\mathrm{mm}^{3}$ cubes with a razor blade and incubated with PBS containing collagenase (Sigma) for 15 minutes at $37^{\circ} \mathrm{C}$. The cell suspension was centrifuged for 5 minutes at 1500 rpm. The supernatant was discarded, and the pellet was resuspended in a complete medium. The isolated cells were plated onto 12-mm glass coverslips in 24-well plates and incubated overnight. The cells were washed with PBS, fixed in cold methanol for 5 minutes, and incubated with a permeabilization buffer $(0.1$ M PIPES [pH 6.9], $1 \mathrm{~mm}$ EGTA, $4 \mathrm{M}$ glycerol, 0.5\% Triton X-100, and $1 \mathrm{~mm}$ GTP) for 1 minute. The cells were then incubated with a blocking solution (10\% normal goat serum, 3\% bovine serum albumin, and $0.5 \%$ gelatin in PBS) for 15 minutes and incubated with primary antibody specific for $\gamma$-tubulin (Sigma), pericentrin (Covance, Richmond, California), or $\alpha$-tubulin (Amersham, Buckinghamshire, United Kingdom) for 1 hour at $20^{\circ} \mathrm{C}$. The antibody-antigen complexes were detected by incubation for 1 hour at $20^{\circ} \mathrm{C}$ with secondary antibodies including Alexa 488-conjugated goat anti-mouse lgG, Alexa 488-conjugated goat anti-rabbit lgG, Alexa 546-conjugated goat anti-mouse IgG, and Alexa 546conjugated goat anti-rabbit IgG (Molecular Probes, Eugene, Oregon). Nuclear DNA was counterstained with 4', 6'-diamidino-2-phenylindole (DAPI) or propidium iodide (PI). Slides were mounted with Gel/Mount (Biomeda Corporation, Foster City, California) and visualized under a laser-scanning microscope (LSM-GB200 System; Olympus, Tokyo, Japan). At least 200 cells in each sample were carefully examined for centrosomes and mitotic spindles. Centrosome numbers were considered abnormal when more than two centrosomes per cell were present.

\section{Fluorescence In Situ Hybridization}

Chromosome numbers were determined by FISH analysis of interphase cells with centromeric probes specific for human chromosomes 1 and 8 . Cells fixed on slides were denatured at $75^{\circ} \mathrm{C}$ for 2 minutes in $70 \%$ formamide/25 SSC (15 SSC $=0.15 \mathrm{M} \mathrm{NaCl}$, $0.015 \mathrm{M}$ sodium citrate), immersed in $70 \%$ ethanol at $-20^{\circ} \mathrm{C}$, and dehydrated in $100 \%$ ethanol. Denatured, biotin-labeled DNA probe specific for the centromeric region of human chromosome 1 or 8 was added to the slides and incubated overnight at $37^{\circ} \mathrm{C}$ in a humid chamber. After hybridization, each slide was washed sequentially for 10 minutes with $50 \%$ formamide at $37^{\circ} \mathrm{C}, 25 \mathrm{SSC}, 15 \mathrm{SSC}$, and $45 \mathrm{SSC}$ at room temper- 

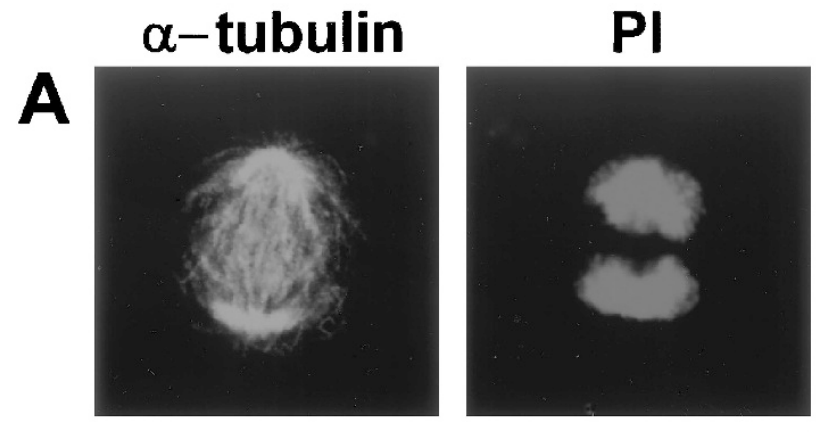

merge
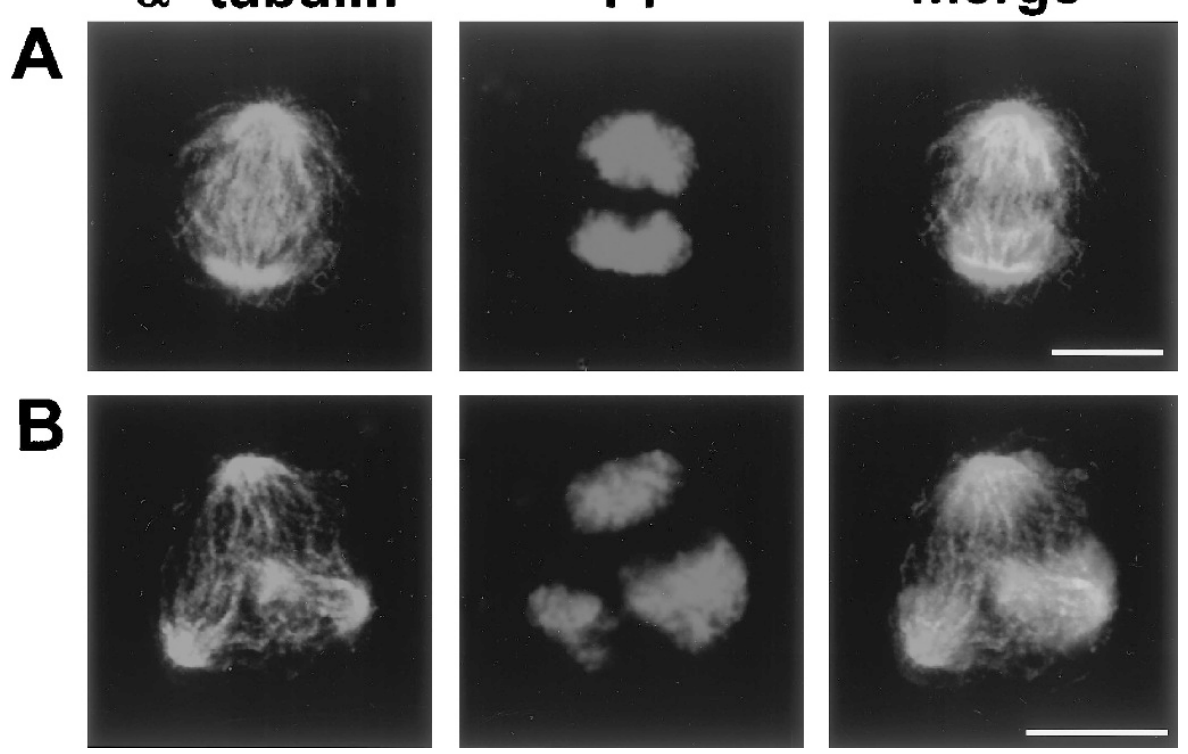

Figure 6.

Chromosome segregation in parental Suit-2 cells and cells isolated from liver metastases. A, Telophase Suit-2 cell shows normal chromosome division. B, Telophase cells from the liver metastasis undergoing multipolar divisions and segregation of chromosomes unequally into three progeny. Scale bars, $10 \mu \mathrm{m}$.

\section{A}

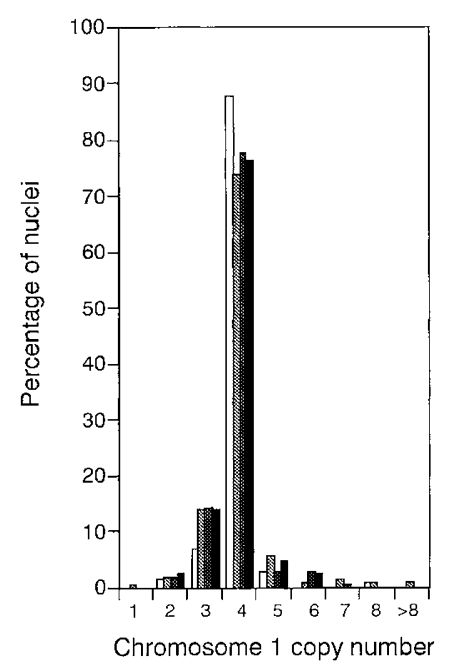

B

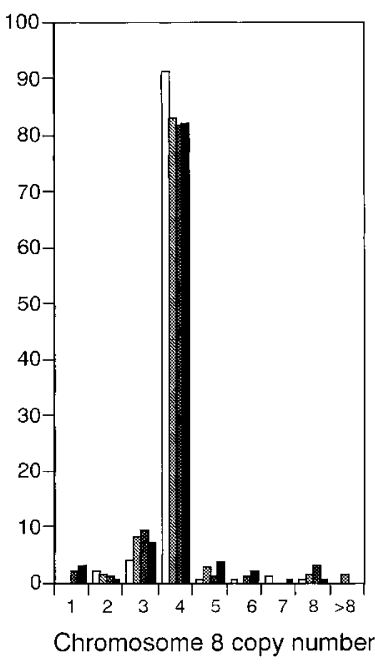

$\square$ Parental Cell

Pancreatic Xenograft

Peritoneal Dissemination

Liver Metastasis

Figure 7.

Chromosome instability in parental Suit-2 cells and cells isolated from pancreatic xenografts and metastatic tumors. Fluorescence in situ hybridization analysis was performed with probes specific for chromosomes 1 (A) and 8 (B), and at least 200 interphase nuclei were counted to determine chromosome copy numbers.

ature, and incubated in 45 SSC/1\% BSA containing avidin-FITC at $37^{\circ} \mathrm{C}$ for 45 minutes. Slides were washed for 5 minutes each in 45 SSC, 45 SSC/0.1\% Triton X-100, 45 SSC, and 25 SSC at room temperature, and counterstained with PI. For each sample, at least 200 interphase cells were examined for chromosome number under a fluorescence microscope (Eclipse E600; Nikon, Tokyo, Japan). For all samples, the modal chromosome numbers and variant fractions (the fraction of cells in which the chromosome number differed from the mode) were determined for each chromosome.

\section{Statistical Analysis}

Statistical significance was evaluated by analysis of variance (ANOVA) or Fisher's exact probability test. Spearman's rank correlation analysis was used to evaluate the relation between the fraction of centrosome defects and the fraction of spindle abnormalities. $p<0.05$ was considered significant.

\section{Acknowledgements}

We thank Ms. Shoko Nishio for technical assistance. 


\section{References}

Carroll PE, Okuda M, Horn HF, Biddinger P, Stambrook PJ, Gleich LL, Li YQ, Tarapore P, and Fukasawa K (1999). Centrosome hyperamplification in human cancer: Chromosome instability induced by p53 mutation and/or Mdm2 overexpression. Oncogene 18:1935-1944.

Dictenberg JB, Zimmerman W, Sparks CA, Young A, Vidair C, Zheng Y, Carrington W, Fay FS, and Doxsey SJ (1998). Pericentrin and $\gamma$-tubulin form a protein complex and are organized into a novel lattice at the centrosome. J Cell Biol 141:163-174.

Doxsey S (1998). The centrosome-a tiny organelle with big potential. Nat Genet 20:104-106.

Doxsey SJ, Stein P, Evans L, Calarco PD, and Kirschner M (1994). Pericentrin, a highly conserved centrosome protein involved in microtubule organization. Cell 76:639-650.

Fu X, Guadagni F, and Hoffman RM (1992). A metastatic nude-mouse model of human pancreatic cancer constructed orthotopically with histologically intact patient specimens. Proc Natl Acad Sci USA 89:5645-5649.

Griffin CA, Hruban RH, Morsberger LA, Ellingham T, Long PP, Jaffee EM, Hauda KM, Bohlander SK, and Yeo CJ (1995). Consistent chromosome abnormalities in adenocarcinoma of the pancreas. Cancer Res 55:2394-2399.

Hahn SA, Seymour AB, Hoque AT, Schutte M, da Costa LT, Redston MS, Caldas C, Weinstein CL, Fischer A, Yeo CJ, Hruban RH, and Kern SE (1995). Allelotype of pancreatic adenocarcinoma using xenograft enrichment. Cancer Res 55:4670-4675.

Hilgers W and Kern SE (1999). Molecular genetic basis of pancreatic adenocarcinoma. Genes Chromosomes Cancer 26:1-12.

Hruban RH, Goggins M, Parsons J, and Kern SE (2000). Progression model for pancreatic cancer. Clin Cancer Res 6:2969-2972.

Iwamura T, Katsuki T, and Ide K (1987). Establishment and characterization of a human pancreatic cancer cell line (SUIT-2) producing carcinoembryonic antigen and carbohydrate antigen 19-9. Jpn J Cancer Res 78:54-62.

Kellogg DR, Moritz M, and Alberts BM (1994). The centrosome and cellular organization. Ann Rev Biochem 63:639674.

Kuo KK, Sato N, Mizumoto K, Maehara N, Yonemasu H, Ker CG, Sheen PC, and Tanaka M (2000). Centrosome abnormalities in human carcinomas of the gallbladder and intrahepatic and extrahepatic bile ducts. Hepatology 31:59-64.

Landis SH, Murray T, Bolden S, and Wingo PA (1999). Cancer statistics, 1999. Ca Cancer J Clin 49:8-31.

Lengauer C, Kinzler KW, and Vogelstein B (1998). Genetic instabilities in human cancers. Nature 396:643-649.

Levine DS, Sanchez CA, Rabinovitch PS, and Reid BJ (1991). Formation of the tetraploid intermediate is associated with the development of cells with more than four centrioles in the elastase-simian virus 40 tumor antigen transgenic mouse model of pancreatic cancer. Proc Natl Acad Sci USA 88: 6427-6431.
Lingle WL, Lutz WH, Ingle JN, Maihle NJ, and Salisbury JL (1998). Centrosome hypertrophy in human breast tumors: Implications for genomic stability and cell polarity. Proc Natl Acad Sci USA 95:2950-2955.

Loeb LA (1991). Mutator phenotype may be required for multistage carcinogenesis. Cancer Res 51:3075-3079.

Pellegata NS, Sessa F, Renault B, Bonato M, Leone BE, Solcia E, and Ranzani GN (1994). K-ras and p53 gene mutations in pancreatic cancer: Ductal and nonductal tumors progress through different genetic lesions. Cancer Res 54: 1556-1560.

Pihan GA and Doxsey SJ (1999). The mitotic machinery as a source of genetic instability in cancer. Semin Cancer Biol 9:289-302.

Pihan GA, Purohit A, Wallace J, Knecht H, Woda B, Quesenberry P, and Doxsey SJ (1998). Centrosome defects and genetic instability in malignant tumors. Cancer Res 58:39743985.

Reyes G, Villanueva A, Garcia C, Sancho FJ, Piulats J, Lluis F, and Capella G (1996). Orthotopic xenografts of human pancreatic carcinomas acquire genetic aberrations during dissemination in nude mice. Cancer Res 56:5713-5719.

Reynolds TY, Rockwell S, and Glazer PM (1996). Genetic instability induced by the tumor microenvironment. Cancer Res 56:5754-5757.

Rofstad EK, Johnsen NM, and Lyng H (1996). Hypoxiainduced tetraploidisation of a diploid human melanoma cell line in vitro. $\mathrm{Br} \mathrm{J}$ Cancer 27:S136-S139.

Sato N, Mizumoto K, Nakamura M, Nakamura K, Kusumoto M, Niiyama H, Ogawa T, and Tanaka M (1999). Centrosome abnormalities in pancreatic ductal carcinoma. Clin Cancer Res 5:963-970.

Sato N, Mizumoto K, Nakamura M, and Tanaka M (2000). Radiation-induced centrosome overduplication and multiple mitotic spindles in human tumor cells. Exp Cell Res 255:321326.

Stearns T, Evans L, and Kirschner M (1991). $\gamma$-tubulin is a highly conserved component of the centrosome. Cell 65: 825-836.

Vezeridis MP, Doremus CM, Tibbetts LM, Tzanakakis G, and Jackson BT (1989). Invasion and metastasis following orthotopic transplantation of human pancreatic cancer in the nude mouse. J Surg Oncol 40:261-265.

Zimmerman W, Sparks CA, and Doxsey SJ (1999). Amorphous no longer: The centrosome comes into focus. Curr Opin Cell Biol 11:122-128.

Zojer N, Fiegl M, Mullauer L, Chott A, Roka S, Ackermann J, Raderer M, Kaufmann H, Reiner A, Huber H, and Drach J (1998). Chromosomal imbalances in primary and metastatic pancreatic carcinoma as detected by interphase cytogenetics: Basic findings and clinical aspects. $\mathrm{Br} \mathrm{J}$ Cancer 77:1337-1342. 